\title{
Hydraulic Model of Dam Break Using Navier Stokes Equation with Arbitrary Lagrangian-Eulerian Approach
}

\author{
Alireza Lohrasbi, Moharram Dolatshahi Pirooz, and Alireza Lavaei
}

\begin{abstract}
The liquid flow and the free surface shape during the initial stage of dam breaking are investigated. A numerical scheme is developed to predict the wave of an unsteady, incompressible viscous flow with free surface. The method involves a two dimensional finite element (2D), in a vertical plan. The Naiver-Stokes equations for conservation of momentum and mass for Newtonian fluids, continuity equation, and full nonlinear kinematic free-surface equation, were used as the governing equations. The mapping developed to solve highly deformed free surface problems common in waves formed during wave propagation, transforms the run up model from the physical domain to a computational domain with Arbitrary Lagrangian Eulerian (ALE) finite element modeling technique.
\end{abstract}

Index Terms-Dam break, naiver-stokes equations, freesurface flows, arbitrary lagrangian eulerian.

\section{INTRODUCTION}

Gravity-driven flows due to dam breaking studied by Zienkiewicz [1], Pohle [2] and Stoker [3] using the Lagrangian description. Pohle presented a systematic procedure for the determination of the successive terms in these expansions. However, only the leading-order terms were constructed and analyzed. In both Lagrangian and Eulerian descriptions the expansions of the solution in time power series should be considered as 'outer' solutions, which are needed to be corrected with 'inner' solutions near the intersection point. However, such an inner solution was successfully derived in a relevant problem concerning a uniformly accelerating wave maker by King and Needham [4]. Several numerical studies performed during the past few years were based on the solution of nonlinear shallow-water equations using different methods such as the finite-volume method, the finite-difference method and so on (see [5]-[10]). There are very analyses of the dam-break problem. Hunt [11] used a kinematic wave approximation to obtain a closedform solution for a sloping channel and mentioned that his solution is valid for large times. The dam-break problem can be interpreted in the context of a liquid column collapsing under gravity [12]. Penney and Thornhill [12] studied the collapse of a fluid column, which is surrounded by a lighter fluid. The analysis was performed in Eulerian variables both for small and moderate times.

They derived the initial asymptotic of the solution for

Manuscript received October 30, 2014; revised February 27, 2015.

Alireza Lohrasbi and Alireza Lavaei are with Department of Civil engineering, College of engineering, Boroujerd Branch, Islamic Azad University, Iran (e-mail: Ar_lohrasbi@yahoo.com, shetab@gmail.com).

Moharram Dolatshahi Pirooz is with School of Civil Engineering, University College of Engineering, University of Tehran, Iran (e-mail: mdolat@ut.ac.ir). fluid columns of semi-cylindrical and hemispherical shapes, and showed that these asymptotic are not valid close to the base, where the fluid velocity is much higher than in the rest of the column.

The numerical solution for free surfaces has been developed using three defined theories: 1) Lagrangian; 2) Eulerian; 3) Arbitrary Lagrangian-Eulerian description. For waves in particular, the Arbitrary Lagrangian-Eulerian is superior in terms of handling high distortion in the grids. To have a versatile description of the fluid domain, it is necessary to have a method with the benefits of both Lagrangian and Eulerian descriptions, without their deficiencies. Such a method, developed in the last two decades, is the "Arbitrary Lagrangian- Eulerian formulation" in which grid points may be moved with the fluid in normal Lagrangian description. This method allows the grids move independent of the fluid motion.

\section{PROBLEM FORMULATION}

We consider the plane problem of gravity-driven flow, which is generated when a vertical dam in front of a liquid region is suddenly removed. Initially condition of the liquid is in 0 .

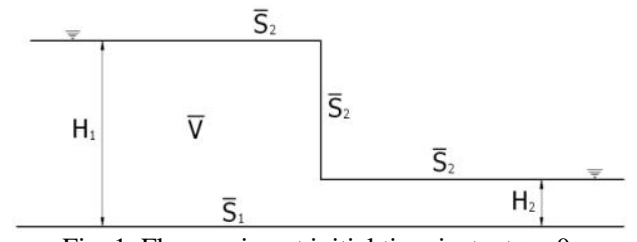

Fig. 1. Flow region at initial time instant $t=0$.

At the initial time instant, $t=0$, the dam is instantly removed and the gravity-driven flow starts. The resulting flow is potential and two-dimensional. We shall determine the liquid flow and the shape of its free surface during the early stages of the process.

The physical domain $V$ surrounded by a piecewise smooth boundary $S$ is shown in 0This domain is occupied by a viscous incompressible fluid with the coefficient of constant kinematic viscosity of $v$ and the specific mass of $\rho$. The problem under consideration is the unsteady motion of a surface wave under gravity. Two-dimensional unsteady incompressible viscous flow is considered. The governing equations are expressed by the unsteady Navier-Stokes equation and the equation of continuity. Let the rectangular coordinates be denoted by $x, y$ and the corresponding velocity components be denoted by $u, v$. As a result, the equations of conservation of momentum and mass, for incompressible Newtonian fluids, in the arbitrary 
Lagrangian-Eulerian form are given as follows:

$$
\begin{aligned}
& \left.\frac{\partial \bar{u}}{\partial \bar{t}}\right|_{\xi, \eta}+\left(\bar{u}-\bar{w}_{u}\right) \frac{\partial \bar{u}}{\partial \bar{x}}+\left(\bar{v}-\bar{w}_{v}\right) \frac{\partial \bar{u}}{\partial \bar{y}}= \\
& -\frac{1}{\rho} \frac{\partial \bar{p}}{\partial \bar{x}}+\bar{v}\left(\frac{\partial^{2} \bar{u}}{\partial \bar{x}^{2}}+\frac{\partial^{2} \bar{u}}{\partial \bar{y}^{2}}\right) \\
& \left.\frac{\partial \bar{v}}{\partial \bar{t}}\right|_{\xi, \eta}+\left(\bar{u}-\bar{w}_{u}\right) \frac{\partial \bar{v}}{\partial \bar{x}}+\left(\bar{v}-\bar{w}_{v}\right) \frac{\partial \bar{v}}{\partial \bar{y}}= \\
& -\frac{1}{\rho} \frac{\partial \bar{p}}{\partial \bar{y}}+\bar{v}\left(\frac{\partial^{2} \bar{v}}{\partial \bar{x}^{2}}+\frac{\partial^{2} \bar{v}}{\partial \bar{y}^{2}}\right)-\bar{g} \\
& \frac{\partial \bar{u}}{\partial \bar{x}}+\frac{\partial \bar{v}}{\partial \bar{y}}=0
\end{aligned}
$$

where $w_{u}$ and $w_{v}$ are the mesh velocities in $\mathrm{x}$ and $\mathrm{y}$ directions.

The boundary $\bar{S}$ consists of two types of boundaries: one is the $\bar{S}_{1}$ on which velocity is given; the other is the free surface boundary $\bar{S}_{2}$ on which the surface force is specified. The boundary conditions can be expressed as the followings,

$$
\begin{gathered}
\bar{u}=\hat{\bar{u}} \text { on } \quad \bar{S}_{1} \\
\bar{v}=\hat{\bar{v}} \text { on } \quad \bar{S}_{1} \\
\left(-\frac{1}{\bar{\rho}} \bar{p}+2 \overline{\bar{v}} \frac{\partial \bar{u}}{\partial \bar{x}}\right) \cdot n_{\bar{x}}+ \\
\bar{v}\left(\frac{\partial \bar{u}}{\partial \bar{y}}+\frac{\partial \bar{v}}{\partial \bar{x}}\right) \cdot n_{\bar{y}}=\hat{\bar{c}}_{x} \\
\text { on } \quad \bar{S}_{2} \\
\bar{v}\left(\frac{\partial \bar{u}}{\partial \bar{y}}+\frac{\partial \bar{v}}{\partial \bar{x}}\right) \cdot n_{\bar{x}}+ \\
\left(-\frac{1}{\bar{\rho}} \bar{p}+2 \bar{v} \frac{\partial \bar{v}}{\partial \bar{y}}\right) \cdot n_{\bar{y}}=\hat{\bar{c}}_{y} \text { on } \quad \bar{S}_{2}
\end{gathered}
$$

where the superscript caret denotes a function which is given on the boundary and $n_{x}$ and $n_{y}$ symbolize the direction cosines of the outward normal to the boundary with respect to co-ordinate $x$ and $y$. Top Equations can be rendered dimensionless by introducing the following variables:

$$
\begin{gathered}
\bar{x}=x \bar{d} \quad \bar{u}=u(\bar{g} \bar{d})^{1 / 2} \\
\bar{y}=y \bar{d} \quad \bar{v}=v(\overline{\bar{g}} \bar{d})^{1 / 2} \\
\bar{p}=p \bar{\rho} \bar{g} \bar{d} \quad \bar{t}=t\left(\frac{\bar{d}}{\bar{g}}\right)^{1 / 2} \\
\left.\frac{\partial u}{\partial t}\right|_{\xi, \eta}+\left(u-w_{u}\right) \frac{\partial u}{\partial x}+\left(v-w_{v}\right) \frac{\partial u}{\partial y}= \\
-\frac{\partial p}{\partial x}+\frac{1}{\operatorname{Re}}\left(\frac{\partial^{2} u}{\partial x^{2}}+\frac{\partial^{2} u}{\partial y^{2}}\right) \\
\left.\frac{\partial v}{\partial t}\right|_{\xi, \eta}+\left(u-w_{u}\right) \frac{\partial v}{\partial x}+\left(v-w_{v}\right) \frac{\partial v}{\partial y}= \\
-\frac{\partial p}{\partial y}+\frac{1}{\operatorname{Re}}\left(\frac{\partial^{2} v}{\partial x^{2}}+\frac{\partial^{2} v}{\partial y^{2}}\right)-1 \\
\frac{\partial u}{\partial x}+\frac{\partial v}{\partial y}=0
\end{gathered}
$$

Using these transformations, the Eq. (1) and Eq. (2) are modified as follows:

\section{NUMERICAL ANALYSIS}

The numerical model is based on a finite element method for the spatial discretization of partial differential equations. This method is implemented using weighted residual variational technique for the solution approach within each element.

\section{A. Basic Concept}

In the temporal discretization, the total time $t$ is divided into a number of short time increments $\Delta t$. Each time point is denoted by $n$. Velocity and pressure at the $n$th time point can be defined as:

$$
\begin{aligned}
& u^{n}=u\left(x, y, t^{n}\right)=u\left(\xi, \eta, t^{n}\right) \\
& v^{n}=v\left(x, y, t^{n}\right)=v\left(\xi, \eta, t^{n}\right) \\
& p^{n}=p\left(x, y, t^{n}\right)=p\left(\xi, \eta, t^{n}\right) \\
& h^{n}=h\left(x, t^{n}\right)=h\left(\xi, t^{n}\right)
\end{aligned}
$$

where $x$ and $y$ denote the coordinate at the $n$th time point in the physical domain. The parameters $\xi$ and $\eta$ are the fixed coordinate at the $n$th time point in the reference domain. Velocity and pressure at time point $n+1$ can be defined subsequently as:

$$
\begin{aligned}
& u^{n+1}=u\left(x, y, t^{n+1}\right)=u\left(\xi, \eta, t^{n+1}\right) \\
& v^{n+1}=v\left(x, y, t^{n+1}\right)=v\left(\xi, \eta, t^{n+1}\right) \\
& p^{n+1}=p\left(x, y, t^{n+1}\right)=p\left(\xi, \eta, t^{n+1}\right) \\
& h^{n+1}=h\left(x, t^{n+1}\right)=h\left(\xi, t^{n+1}\right)
\end{aligned}
$$

In the Eulerian treatment, the spatial differentiation can be approximated in the form:

$$
\begin{gathered}
\left.\frac{\partial u}{\partial t}\right|_{\xi, \eta}=\frac{u^{n+1}-u^{n}}{\Delta t} \\
\left.\frac{\partial v}{\partial t}\right|_{\xi, \eta}=\frac{v^{n+1}-v^{n}}{\Delta t} \\
\left.\frac{\partial h}{\partial t}\right|_{\xi, \eta}=\frac{h^{n+1}-h^{n}}{\Delta t} \\
\frac{u^{n+1}-u^{n}}{\Delta t}=-\left(u^{n}-w_{u}{ }^{n}\right) \frac{\partial u^{n}}{\partial x}- \\
\left(v^{n}-w_{v}{ }^{n}\right) \frac{\partial u^{n}}{\partial y}-\frac{\partial p^{n+1}}{\partial x}+ \\
\frac{1}{\operatorname{Re}}\left(\frac{\partial^{2} u^{n}}{\partial x^{2}}+\frac{\partial^{2} u^{n}}{\partial y^{2}}\right) \\
\frac{v^{n+1}-v^{n}}{\Delta t}=-\left(u^{n}-w_{u}{ }^{n}\right) \frac{\partial v^{n}}{\partial x}- \\
\left(v^{n}-w_{v}{ }^{n}\right) \frac{\partial v^{n}}{\partial y}-\frac{\partial p^{n+1}}{\partial x}+ \\
\frac{1}{\operatorname{Re}}\left(\frac{\partial^{2} v^{n}}{\partial x^{2}}+\frac{\partial^{2} v^{n}}{\partial y^{2}}\right)-1 \\
\frac{\partial u^{n+1}}{\partial x}+\frac{\partial v^{n+1}}{\partial y}=0 \\
\frac{h^{n+1}-h^{n}}{\Delta t}=-\left(u^{n+1}-w_{u}{ }^{n+1}\right) \frac{\partial h^{n}}{\partial x}+v^{n+1}
\end{gathered}
$$


With substituting Equations, the equations of motion, continuity and kinematic boundary condition can be discredited into,

The boundary conditions corresponding are described by:

$$
\begin{gathered}
u^{n+1}=\hat{u} \\
v^{n+1}=\hat{v} \\
\left(-p^{n+1}+\frac{2}{\operatorname{Re}} \frac{\partial u^{n+1}}{\partial x}\right) \cdot n_{x}+ \\
\frac{1}{\operatorname{Re}}\left(\frac{\partial u^{n+1}}{\partial y}+\frac{\partial v^{n+1}}{\partial x}\right) \cdot n_{y}=\hat{c}_{x} \\
\frac{1}{\operatorname{Re}}\left(\frac{\partial u^{n+1}}{\partial y}+\frac{\partial v^{n+1}}{\partial x}\right) \cdot n_{x}+ \\
\left(p^{n+1}+\frac{2}{\operatorname{Re}} \frac{\partial v^{n+1}}{\partial y}\right) \cdot n_{y}=\hat{c}_{y}
\end{gathered}
$$

The analysis procedure presented here involves computing the unknown variable $u^{n+1}, v^{n+1}, p^{n+1}, h^{n+1}$ and the boundary conditions, starting from the known variable $u^{n}$, $v^{n}, p^{n}, h^{n}$.

To solve Equations, the fractional method is employed. This method is one of the earliest and the most widely used method for solving fluid dynamic problems. In this method, by discretizing the equations of motion, the intermediate velocity can be obtained. However, this velocity may not satisfy the equation of continuity. To correct the obtained intermediate velocity, a correction potential should be introduced. The Poisson equation for the correction potential can be derived by trying to satisfy the equation of continuity. By solving the resultant Poisson equation, the correction velocity vector can be obtained.

$$
\begin{aligned}
& \frac{\tilde{u}^{n+1}-u^{n}}{\Delta t}=-\left(u^{n}-w_{u}{ }^{n}\right) \frac{\partial u^{n}}{\partial x}- \\
& \left(v^{n}-w_{v}{ }^{n}\right) \frac{\partial u^{n}}{\partial y}-\frac{\partial p^{n+1}}{\partial x}+ \\
& \frac{1}{\operatorname{Re}}\left(\frac{\partial^{2} u^{n}}{\partial x^{2}}+\frac{\partial^{2} u^{n}}{\partial y^{2}}\right) \\
& \frac{\tilde{v}^{n+1}-v^{n}}{\Delta t}=-\left(u^{n}-w_{u}{ }^{n}\right) \frac{\partial v^{n}}{\partial x}- \\
& \left(v^{n}-w_{v}{ }^{n}\right) \frac{\partial v^{n}}{\partial y}-\frac{\partial p^{n+1}}{\partial x}+ \\
& \frac{1}{\operatorname{Re}}\left(\frac{\partial^{2} v^{n}}{\partial x^{2}}+\frac{\partial^{2} v^{n}}{\partial y^{2}}\right)-1 \\
& \frac{\partial u^{n+1}}{\partial y}-\frac{\partial v^{n+1}}{\partial x}=\frac{\partial \tilde{u}^{n+1}}{\partial y}-\frac{\partial \tilde{v}^{n+1}}{\partial x}
\end{aligned}
$$

This equation implies that

$$
u^{n+1}=\tilde{u}^{n+1}+\frac{\partial \phi}{\partial x} \quad v^{n+1}=\tilde{v}^{n+1}+\frac{\partial \phi}{\partial x}
$$

where $\phi$ is a scalar which is referred to as the correction potential. By taking the partial derivation on both sides with regard to $x$ and $y$ respectively and adding them together, we have

$$
\begin{aligned}
& \frac{\partial u^{n+1}}{\partial x}+\frac{\partial v^{n+1}}{\partial y}= \\
& \frac{\partial \tilde{u}^{n+1}}{\partial x}+\frac{\partial \tilde{v}^{n+1}}{\partial y}+\frac{\partial^{2} \phi}{\partial x^{2}}+\frac{\partial^{2} \phi}{\partial y^{2}}
\end{aligned}
$$

With substitutingthe equation of continuity, the equation for $\phi$ can be derived as

$$
\frac{\partial^{2} \phi}{\partial x^{2}}+\frac{\partial^{2} \phi}{\partial y^{2}}=-\frac{\partial \tilde{u}^{n+1}}{\partial x}-\frac{\partial \tilde{v}^{n+1}}{\partial y}
$$

With substituting, the equation of controlling the evolution of pressure can be expressed

$$
\begin{aligned}
& \frac{\partial p^{n+1}}{\partial x}=\frac{\partial p^{n}}{\partial x}-\frac{1}{\Delta t} \frac{\partial \phi}{\partial x} \\
& \frac{\partial p^{n+1}}{\partial y}=\frac{\partial p^{n}}{\partial y}-\frac{1}{\Delta t} \frac{\partial \phi}{\partial y} \\
& p^{n+1}=p^{n}-\frac{1}{\Delta t} \phi
\end{aligned}
$$

In order to implement a numerical solution procedure for the arbitrary Lagrangian Eulerian formulation, the momentum equation and the incompressibility constraint of the Navier-Stokes problem are analyzed using a procedure which consists of six separate phases. Let $u^{n}, v^{n}, p^{n}$, $h^{n}$ be the velocities, pressure and wave height fields at time $\mathrm{t}^{\mathrm{n}}$, where $t^{n+1}=t^{n}+\Delta t$. From $u^{n}, v^{n}, p^{n}, h^{n}$ and the boundary specifications, the fields $u^{n+1}, v^{n+1}, p^{n+1}$, $h^{n+1}$ are calculated.

\section{TRANSFORMATION OF THE BASIC EQUATIONS INTO THE MAPPED COORDINATE SYSTEM}

The computation of the propagation of free surface waves involves computational boundaries that do not coincide with coordinate lines in physical space. For the finite element method, such problem requires a complicated interpolation function on the local grid lines which results in the local loss of accuracy in the computational solution. Such difficulties require a mapping or transformation from physical space to a generalized space. This transformation simplifies the problem of highly deformed air-fluid interface that arises in the analysis of wave breaking. This mapping transforms the wave propagation model from the physical domain, $(x, y)$ to a computational domain, $(\xi, \eta)$. The use of generalized coordinates implies that a distorted region in physical space, such as breaking wave, is mapped into a rectangular region in the generalized coordinate space, where the unknown interface coincides with a coordinate line as in 0

Since the interior points in the computational domain form a regular grid and the boundaries coincide with coordinate lines, the determination of $x(\xi, \eta), y(\xi, \eta)$ is easier than 
working in the irregular physical domain. With Referring to the physical and computational meshes picture 0 , the following mapping, can be established.

\section{$111,1,1,111111111111$}

Fig. 2. The computational grid is shown mapped back to the physical space.

$$
\begin{aligned}
& x=\sum_{i=1}^{3}\left(\xi+h \alpha_{i}\right) F_{i}(\eta) \\
& y=\eta(1+h)
\end{aligned}
$$

The function $F_{i}(\eta)$ is interpolation function. With Employing three point interpolations as shown in 0, we have:

$$
\begin{aligned}
& F_{1}(\eta)=1-3 \eta+2 \eta^{2} \\
& F_{2}(\eta)=4 \eta-4 \eta^{2} \\
& F_{3}(\eta)=-\eta+2 \eta^{2}
\end{aligned}
$$

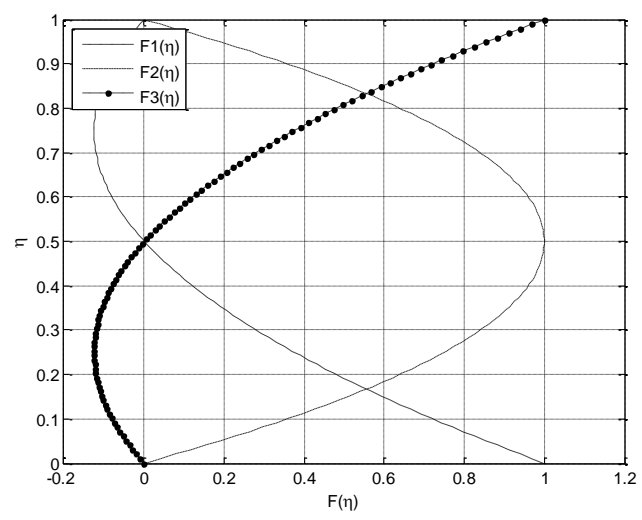

Fig. 3. Three point interpolation function.

\section{A. Eulerian Description}

To have an Eulerian description, where the physical coordinate system coincide with the generalized coordinate system, it is necessary to set $\alpha_{i}=\beta=h=0$.

\section{B. Eulerian Description in $x$ Direction and Lagrangian Description in y Direction}

Eulerian Description in $x$ direction and Lagrangian Description in direction can be applied for nonbreaking waves. In this case, it is necessary to set $\alpha_{i}=0$. The transformation is Lagrangian in $y$ direction and Eulerian in $x$ direction and the problems associated with this transformation should have single value profile.

\section{Arbitrary Lagrangian-Eulerian Description}

The Arbitrary Lagrangian-Eulerian algorithm is employed in modelling wave propagation both over sloping beaches, where the evolution occurs over bathymetry topography, and over constant depth regions. Different types of $\alpha_{i}$ values are provided and depending on the nature of the problem. To coinside physical and computational boundary, the $\alpha_{i}$ values are considered to be a fifth order polynomial function of $\xi$ as followed:

$$
\begin{aligned}
& \alpha_{i}=\frac{b}{\varepsilon^{3} l^{5}(1-\varepsilon)^{3}}\left[2\left(\xi-\xi_{0}\right)^{5}(2 \varepsilon-1)+\right. \\
& l\left(\xi-\xi_{0}\right)^{4}\left(4-5 \varepsilon-5 \varepsilon^{2}\right)+ \\
& 2 l^{2}\left(\xi-\xi_{0}\right)^{3}\left(-1-\varepsilon+5 \varepsilon^{2}\right)+ \\
& \left.l^{3} \varepsilon\left(\xi-\xi_{0}\right)^{2}(3-5 \varepsilon)\right]
\end{aligned}
$$

and

$$
\alpha_{i}=C \alpha_{i-1} \quad 0<C<0 / 5
$$

Definitions of $b, \varepsilon, 1$ and $\xi_{0}$ are illustrated in 0 . Parameter $\mathrm{C}$ is a constant coefficient and its value is obtained by trial and error to stabilize the problem.

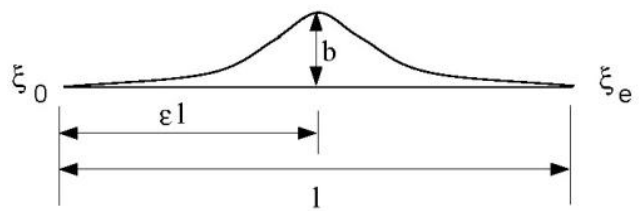

Fig. 4. Definition of parameters in $\alpha_{i}$ function.

\section{Variational Equations in the Transformed Domain}

Spatial discretization of partial differential equations in the numerical model is based on a Galerkin finite element method. This method is implemented using the weighted residual variational method for solution within each element. Using standard linear shape functions for a rectangular element in natural coordinate system, the velocity, pressure and correction potential fields within the element are interpolated in terms of their nodal values as follows:

$$
\begin{aligned}
& u=\psi_{\alpha} u_{\alpha} \\
& v=\psi_{\alpha} v_{\alpha} \\
& p=\psi_{\alpha} p_{\alpha} \\
& \phi=\psi_{\alpha} \phi_{\alpha} \\
& h=\psi_{\alpha s} h_{\alpha}
\end{aligned}
$$

$$
\begin{aligned}
& M_{\alpha \beta}\left|J^{-1}\right|^{n} \tilde{u}_{i \beta}{ }^{n+1}=M_{\alpha \beta}\left|J^{-1}\right|^{n} u_{i \beta}{ }^{n} \\
& -\frac{\Delta t}{\operatorname{Re}}\left[\left(\frac{2}{j} \delta_{i, 1}+j \delta_{i, 2}\right) \xi_{k, j}^{n} \xi_{l, j}^{n} M_{\alpha k \beta l}\right]\left|J^{-1}\right|^{n} u_{i \beta}{ }^{n} \\
& -\frac{\Delta t}{\operatorname{Re}} \xi_{l, j}^{n} \xi_{j, 2}^{n}\left(\delta_{i, 1} M_{\alpha j \beta l} u_{2 \beta}^{n}+\delta_{i, 2} M_{\alpha l \beta j} u_{1 \beta}^{n}\right)\left|J^{-1}\right|^{n} \\
& -\Delta t\left[\xi_{l, j}^{n} M_{\alpha \beta \beta l}\left(u_{j \beta}^{n}-w_{u j \beta}^{n}\right)\right]\left|J^{-1}\right|^{n} u_{i \beta}^{n} \\
& +\Delta t \xi_{j, i}^{n} M_{\alpha j \beta}\left|J^{-1}\right|^{n} p_{\beta}{ }^{n}+\sum_{\xi_{i}}^{n}
\end{aligned}
$$

where $\psi_{\alpha}$ is the interpolation function and $u, v, \phi, h$ represent the nodal values at the node of the $j^{\text {th }}$ element. $\phi$ is a scalar which is referred to as the correction potential base on the Fractional step method presented by Hayashi and hatanaka (1991). By dividing the total time $t$ 
into a number of short time increments $\Delta t$, the equations of motion, continuity and kinematic boundary condition can be discretized into:

where

$$
\begin{array}{ccc}
M_{\alpha}=\int_{V} \psi_{\alpha} d V & M_{\alpha \beta \beta 1}=\int_{V} \psi_{\alpha} \psi_{\beta} \frac{\partial \psi_{\beta}}{\partial \xi} d V \\
M_{\alpha \beta}=\int_{V} \psi_{\alpha} \psi_{\beta} d V & M_{\alpha \beta \beta 2}=\int_{V} \psi_{\alpha} \psi_{\beta} \frac{\partial \psi_{\beta}}{\partial \eta} d V \\
M_{\alpha 1 \beta 1}=\int_{V} \frac{\partial \psi_{\alpha}}{\partial \xi} \frac{\partial \psi_{\beta}}{\partial \xi} d V & H_{\alpha \beta}=\int_{S} \psi_{\alpha} \psi_{\beta} d S \\
M_{\alpha 1 \beta 2}=\int_{V} \frac{\partial \psi_{\alpha}}{\partial \xi} \frac{\partial \psi_{\beta}}{\partial \eta} d V & H_{\alpha \beta 1}=\int_{S} \psi_{\alpha} \frac{\partial \psi_{\beta}}{\partial \xi} d S \\
M_{\alpha 2 \beta 1}=\int_{V} \frac{\partial \psi_{\alpha}}{\partial \eta} \frac{\partial \psi_{\beta}}{\partial \xi} d V & H_{\alpha \beta 2}=\int_{S} \psi_{\alpha} \frac{\partial \psi_{\beta}}{\partial \eta} d S \\
M_{\alpha \beta 1}=\int_{V} \frac{\partial \psi_{\alpha}}{\partial \xi} \psi_{\beta} d V & H_{\alpha \beta \beta 1}=\int_{S} \psi_{\alpha} \psi_{\beta} \frac{\partial \psi_{\beta}}{\partial \xi} d S \\
M_{\alpha \beta 2}=\int_{V} \psi_{\alpha} \frac{\partial \psi_{\beta}}{\partial \eta} d V & H_{\alpha \beta \beta 2}=\int_{S} \psi_{\alpha} \psi_{\beta} \frac{\partial \psi_{\beta}}{\partial \eta} d S
\end{array}
$$

It should be noted that all of the derivations are with respect to $\xi_{i}$.

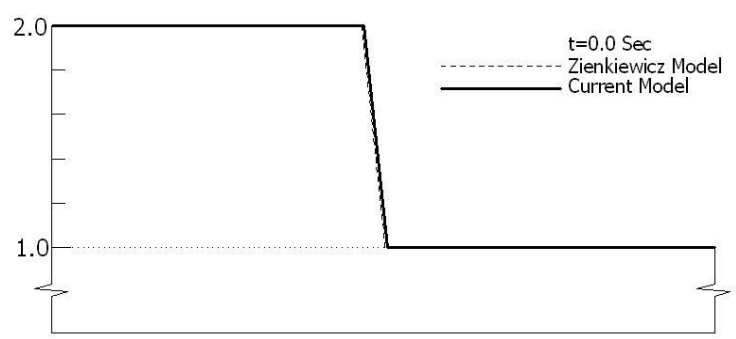

Fig. 5. Dam break model with present numerical model at $\mathrm{t}=0.0$ $(\mathrm{H} 1 / \mathrm{H} 2=2.0)$.

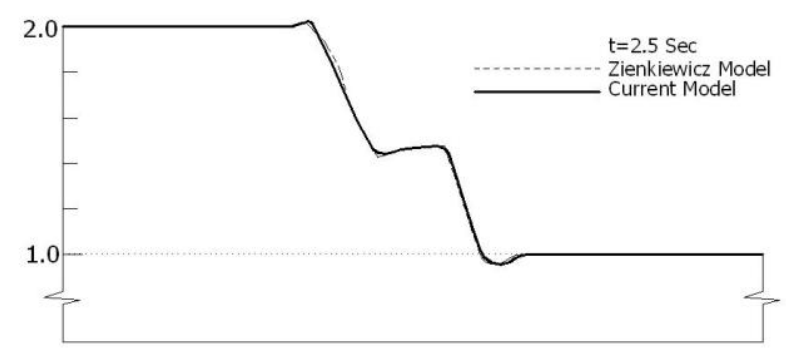

Fig. 6. Dam break model with present numerical model at $\mathrm{t}=2.5$ $(\mathrm{H} 1 / \mathrm{H} 2=2.0)$.

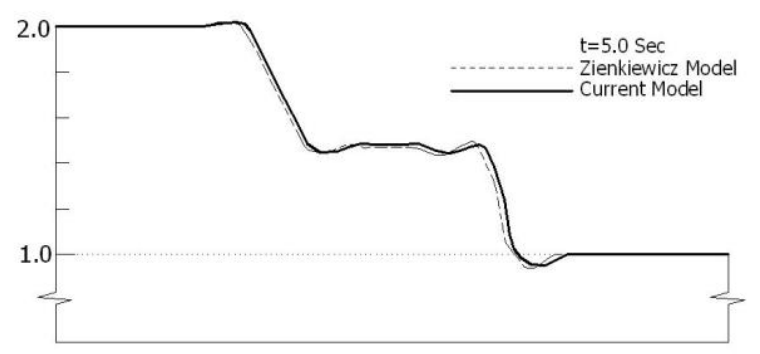

Fig. 7. Dam break model with present numerical model at $\mathrm{t}=5.0$ $(\mathrm{H} 1 / \mathrm{H} 2=2.0)$.

\section{CONCLUSION}

The propagation and deformation of free surface in dam break over flat bathymetry is investigated. Here a dam separating two stationary water levels is suddenly removed and the almost vertical waves progress into the two domains. 0Illustrates 'dam break' problem diagrammatically with $\mathrm{H} 1 / \mathrm{H} 2=2.0$. Oand 0show the dam break at $t=2.5 \mathrm{sec}$ and $t=5 \mathrm{sec}$, respectively.

This model has the capability of dam break model until $\mathrm{H} 1 / \mathrm{H} 2=4.5$. The arbitrary Lagrangian-Eulerian description is examined where the spatial coordinates are moving with the velocity and the computation is done in the reference coordinate system $\xi$ and $\eta$. The reason for the selecting of arbitrary Lagrangian-Eulerian description for modeling of dam breaking wave is force the model to cope with several of wave profiles and the model can be employed in any geometry, under complicated boundary conditions, and with arbitrary bathymetry, without any additional computational effort. The model is validated by comparing numerical results with theoretical solutions and with results obtained numerically. Overall, the conformity between the available data and the computations is well and in most cases the numerical model gives excellent results. The method is tested on a free, steady wave of finite amplitude, and then applied to unsteady waves and is found to give excellent agreement with independent calculations based on the other existing theories.

\section{REFERENCES}

[1] R. L. Zienkiewicz, J. Taylor, and Z. Zhu, The Finite Element Method: Its Basis and Fundamentals, Sixth Edition, 2005.

[2] F. V. Pohle, "The Lagrangian equations of hydrodynamics: solutions which are analytic functions of time," PhD dissertation, New York University, USA, 1950.

[3] J. J. Stoker, Water Waves, Interscience Publishers Inc, New York, 1957.

[4] A. C. King and D. J. Needham, "The initial development of a jet caused by fluid, body and free surface interaction," Journal Fluid Mech, vol. 268, pp. 89-101, 1994.

[5] C. Zoppou and S. Roberts, "Explicit schemes for dam-break simulations," Journal Hydraul Eng, vol. 129, pp. 11-34, 2003.

[6] P. Glaister, "Solutions of a two dimensional dam break problem," Int $J$ Eng Sci, vol. 29, pp. 1357-1362, 1991.

[7] P. Glaister, "Approximate Riemann solutions of the shallow water equations," Journal Hydraul Res, vol. 26, pp. 293-300, 1988.

[8] F. Luigi and E. F. Toro, "Experimental and numerical assessment of the shallow water model for two dimensional dam-break problems," Journal Hydraul Res, vol. 33, pp. 843-864, 1995.

[9] C. Zoppou and S. Roberts, "Numerical solution of the twodimensional unsteady dam break," Appl Math Fluids Res, vol. 24, no. $457-475,2000$.

[10] P. Brufau and P. Garcia-Navarro, "Two dimensional dam break flow simulation," Int J Numer Methods Fluids Res, vol. 33, pp. 55-57, 2000.

[11] B. Hunt, "Asymptotic solution for dam break problem," J Hydraul Div, ASCE, vol. 108, pp. 115-126, 1982.

[12] W. G. Penney and C. K. Thornhill, "The dispersion, under gravity, of a column of fluid supported on a rigid horizontal plane," Phil Trans Roy Soc London A 244, pp. 285-311, 1952.

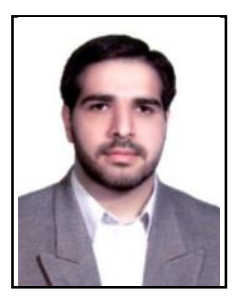

A. Lohrasbi is an academic member in Department of Civil Engineering, College of Engineering, Boroujerd Branch, Islamic Azad University, Iran.

He started teaching civil course in Azad Islamic University since 2006.

His research interests include inundation field surveys, numerical and analytical modeling, and hazard assessment, mitigation and planning, Breaking waves.

$\mathrm{He}$ is a member of American Concrete Institute (ACI) and member of Construction Engineering Disciplinary Organization (Level 1 in Design and Control) in Iran. 